\title{
PERFIL DE CUIDADORES DE IDOSOS COM DOENÇA DE ALZHEIMER ASSOCIADO À RESILIÊNCIA ${ }^{1}$
}

\author{
Cheila Cristina Leonardo de Oliveira Gaioli², Antonia Regina Ferreira Furegato ${ }^{3}$, Jair Lício Ferreira Santos ${ }^{4}$
}

\footnotetext{
${ }^{1}$ Trabalho extraído da tese - Cuidadores de idosos com doença de Alzheimer: variáveis sociodemográficas e da saúde, associadas à resiliência, apresentada ao Curso de Pós- Graduação do Programa Enfermagem Psiquiátrica da Escola de Enfermagem de Ribeirão Preto (EERP) da Universidade de São Paulo (USP), em 2010.

${ }^{2}$ Doutora em Ciências da Saúde. Enfermeira do Departamento de Enfermagem Psiquiátrica e Ciências Humanas da EERP/ USP. São Paulo, Brasil. E-mail: cheila@eerp.usp.br

${ }^{3}$ Doutora em Enfermagem. Professora Titular do Departamento de Enfermagem Psiquiátrica e Ciências Humanas da EERP/ USP. São Paulo, Brasil. E-mail: furegato@eerp.usp.br

${ }^{4}$ Doutor em Saúde Pública. Professor Titular do Departamento de Medicina Social da Faculdade de Medicina de Ribeirão Preto da USP. São Paulo, Brasil. E-mail: jairlfs@fmrp.usp.br
}

RESUMO: Estudo exploratório-descritivo que objetivou descrever variáveis sociodemográficas e de saúde dos cuidadores de idosos com Alzheimer, associando os cuidados realizados à resiliência. Participaram do estudo 101 cuidadores, maiores de 18 anos, que acompanhavam os idosos em unidade básica e em hospital público, no ano 2009. Foram aplicados questionários para perfil, Inventário de Depressão de Beck e Escala de Resiliência. Realizada análise estatística dos dados. A maioria dos cuidadores eram mulheres, sem depressão, recebia ajuda de outras pessoas para cuidar e possuía alto grau de resiliência. Houve associação significativa da resiliência com as variáveis: grau de parentesco, tratamento médico, uso de medicamentos, cansaço, esgotamento, desânimo e saúde mental do cuidador. Saúde física foi associada, significativamente, à experiência no cuidado, sendo que 82 idosos tinham prejuízos cognitivos graves. O idoso no contexto familiar pode ser beneficiado quando o cuidador é mais resiliente.

DESCRITORES: Cuidadores. Doença de Alzheimer. Resiliência psicológica.

\section{PROFILE OF CAREGIVERS OF ELDERLY PEOPLE WITH ALZHEIMER DISEASE ASSOCIATED TO RESILIENCE}

\begin{abstract}
This exploratory and descriptive study described sociodemographic and health variables of caregivers of elderly people with Alzheimer's disease, associating care provided with resilience. Participants were 101 caregivers over 18 years old who accompanied older adults in a Primary Care Unit of a Brazilian public hospital in 2009. Questionnaires regarding the profile, the Beck Depression Inventory, and the Resilience Scale were used. Descriptive statistical analysis was performed. Most caregivers were female, without depression, aided by other people in providing care, and had a high degree of resilience. The variables degree of kinship, medical treatment, the use of medication, tiredness, prostration, discouragement, and caregivers' mental health had significant association with resilience. Physical health was significantly associated to experience in care, with 82 elderly people presenting acute cognitive damage. Older adults in the family context can benefit from a more resilient caregiver.
\end{abstract}

DESCRIPTORS: Caregivers. Alzheimer disease. Resilience, psychological.

\section{PERFIL DE CUIDADORES DE ANCIANOS CON ENFERMEDAD DE ALZHEIMER ASOCIADO CON RESILIENCIA}

\begin{abstract}
RESUMEN: Este estudio exploratorio y descriptivo objetivó describir variables sociodemográficas y de salud de los cuidadores de ancianos con Alzheimer, asociando la atención prestada con la resiliencia. Participaron 101 cuidadores, mayores de 18 años, que acompañaban los ancianos en un Servicio de Salud y en un hospital público, en 2009. Fueron aplicados cuestionarios para perfil, el Inventario de Depresión de Beck y la Escala de Resiliencia, con análisis descriptivo de los datos. La mayoría de los cuidadores eran mujeres, sin depresión, recibían ayuda de otras personas para cuidar y tenían alto grado de resiliencia. Las variables grado de parentesco, tratamiento médico, uso de medicamentos, cansancio, agotamiento, desánimo y salud mental del cuidador tuvieron asociación significativa con resiliencia. Salud física fue significativamente asociada con experiencia en el cuidado, siendo que 82 ancianos tenían prejuicios cognitivos graves. Ancianos en el contexto familiar pueden ser beneficiados por cuidadores con más resiliencia.
\end{abstract}

DESCRIPTORES: Cuidadores. Enfermedad de Alzheimer. Resiliencia psicológica. 


\section{INTRODUÇÃO}

O índice de envelhecimento no Brasil aponta para mudanças na estrutura etária da população. Em 2008, para cada grupo de 100 crianças de zero a 14 anos existiam 24 idosos de 65 anos ou mais. Em 2050, o quadro mudará e para cada 100 crianças de zero a 14 anos existirão 172 idosos. ${ }^{1}$ Esse processo aponta para crescente complexidade de determinantes e de estratégias alternativas para atender as necessidades dessa população.

Do ponto de vista da saúde dos idosos, destacam-se mudanças significativas no quadro de morbimortalidade, isto é, diminuição na incidência e morte por doenças infectocontagiosas e aumento da incidência e morte por doenças crônicas degenerativas, típicas de idades mais avançadas. Esse quadro requer reorganização estrutural da assistência que se prolongará por mais tempo e a necessidade de cuidadores aumentará.

Ao considerar o papel do cuidador de idosos, tanto familiar quanto profissional, existe tendência predominante de investigação dos efeitos negativos nessa função, com destaque para as doenças físicas, psicossomáticas, ansiedade, depressão e estresse. Entretanto, nem todos os cuidadores desenvolvem doenças ou se tornam insatisfeitos com a tarefa de cuidar. Isso pode ser explicado com a utilização de diferentes estratégias individuais para lidar com as situações consideradas desgastantes.

Por isso, busca-se conhecer melhor os fatores resilientes das pessoas como forma de superar ou ressignificar os problemas cotidianos. A resiliência é conceituada como conjunto de processos sociais e intrapsíquicos que possibilitam o desenvolvimento de vida sadia, mesmo vivendo em um ambiente não sadio. ${ }^{2}$

Não foram encontrados trabalhos na enfermagem brasileira que enfoquem especificamente o tema resiliência em associação ao perfil de cuidadores de idosos, com doença de Alzheimer. Entretanto, acredita-se que a resiliência seja um conceito que pode ser significativo para o redimensionamento das pesquisas em enfermagem, contribuindo para reflexões na área do ensino e das práticas de cuidado. ${ }^{3}$

A atividade de cuidar de pessoas demenciadas pode gerar, ao longo desse processo, desgaste para o cuidador, profissional ou leigo e comprometer a sua condição de saúde física e mental, levando a quadros de ansiedade, depressão e distúrbios de comportamento. ${ }^{4}$
A progressão da doença é variável, o que possibilita caracterizar as fases do processo demencial em inicial, intermediária e avançada, mesmo com as diferenças individuais. As manifestações da doença de Alzheimer levam a múltiplas demandas para o cuidador, o que torna o cuidado tarefa difícil de se realizar, pois o indivíduo doente precisará de cuidados constantes e cada vez mais complexos.

Com isso, as necessidades de cuidado extrapolam, muitas vezes, a capacidade das famílias e cresce, portanto, a necessidade de cuidadores formais, com capacitação profissional para o cuidado com o idoso. Organizações internacionais e a política nacional apontam para a necessidade da formação de profissionais capacitados para lidarem com as pessoas idosas. ${ }^{5}$ Porém, o cuidado com o idoso geralmente acontece no domicílio e, frequentemente, quem cuida é um familiar, o que justifica a necessidade de maiores investimentos no preparo dessas pessoas para conseguirem realizar o cuidado mais adequado dentro de suas possibilidades.

O enfermeiro, como parte da equipe de saúde, pode oferecer aos cuidadores subsídios para a instrumentalização do cuidado e orientar a adaptação dos mesmos no contexto domiciliar, referente à progressiva dependência do idoso consequente à doença de Alzheimer.

Apesar dos problemas e dificuldades enfrentados na tarefa de cuidar de idosos de longa dependência, existe a possibilidade de resultados emocionais positivos para esses cuidadores, os quais podem estar associados a maior ou menor resiliência. Assim, este estudo objetivou caracterizar o perfil dos cuidadores de idosos com doença de Alzheimer, os cuidados que realizam, e associá-lo à resiliência.

\section{MÉTODO}

Realizou-se estudo exploratório-descritivo em um ambulatório de hospital geral e Unidade Básica de Saúde de Ribeirão Preto, SP, do Sistema Único de Saúde (SUS). Esses serviços de saúde prestam um atendimento semanal, reconhecidos como centro de pesquisa e referência para diagnóstico de demência, com média de 15 casos, entre retornos e casos novos. O projeto foi aprovado por Comitê de Ética em Pesquisa dessas instituições, conforme normas exigidas pela Resolução ${ }^{\circ}$ 196/96, do Conselho Nacional de Saúde (Processo HCRP n¹1827/08). 
A amostra foi constituída por 101 cuidadores, que atendiam aos critérios de seleção: ser acompanhantes do idoso nesses serviços, maiores de 18 anos, que exerciam a função de cuidador com o mesmo idoso, há, pelo menos, um ano. Aplicaram-se os questionários, com leitura das perguntas e registro das respostas dos cuidadores, pela pesquisadora. Os cuidadores foram convidados no dia do retorno do familiar adoecido e mediante seu aceite foi realizada a entrevista em sala reservada.

Os idosos tinham, em seus prontuários, o diagnóstico confirmado da doença de Alzheimer e resultados do Clinical Dementia Rating (CDR) para mensurar estadiamento da demência e Mini Exame do Estado Mental (MEEM) para mensurar prejuízo cognitivo. Foram considerados os resultados desses testes dentro de um período de seis meses.

Para a coleta dos dados, em relação ao idoso, investigou-se apenas idade, sexo, escolaridade e resultado do MEEM e CDR. Referente ao cuidador investigou-se o perfil sociodemográfico, de saúde e dados do cuidado prestado, utilizando-se questionário com questões fechadas elaborado pelos pesquisadores com coleta de informações sociodemográficas dos idosos e dos cuidadores, além de dados sobre a saúde, hábitos de vida e do cuidado prestado pelo cuidador. Além disso, foram utilizados dois instrumentos validados, o Inventário de Depressão de Beck ${ }^{6}$, em forma de escala com 21 itens, amplamente aplicado em pesquisas clínicas ou de rastreamento de sintomatologia depressiva e a Escala de Resiliência, desenvolvida primeiramente por Wagnild e Young. ${ }^{7}$ Esse é um dos poucos instrumentos usados para medir níveis de adaptação psicossocial positiva em face de eventos importantes de vida e foi utilizado para adaptação transcultural, confiabilidade e validação para a Escala de Resiliência ${ }^{8}$ na língua portuguesa, utilizada nesse estudo. Essa escala é composta por 25 itens descritos de forma positiva com resposta tipo likert, variand o de 1 (discordo totalmente) a 7 (concordo totalmente) e os escores da escala oscilam de 25 a 175 pontos, com valores altos indicando elevada resiliência. Foram destacados bons resultados da validade de constructo, indicando capacidade para discriminar vários atributos relacionados à resiliência, entre eles, correlação direta e significativa com autoestima, satisfação de vida e apoio social.

A coleta dos dados ocorreu no período de fevereiro de 2009 a janeiro de 2010.

Os dados foram organizados em planilha do Excel e os resultados referentes à identificação dos sujeitos, dados de saúde e do cuidado foram sub- metidos à análise estatística descritiva e algumas variáveis foram associadas à Escala de Resiliência (ER), pelo Teste Exato de Fisher, utilizando-se o Stata. O resultado do escore final da ER foi analisado em tercis (baixo, médio e alto). Os resultados foram discutidos com base na literatura científica sobre o tema.

\section{RESULTADOS}

Os resultados analisados neste estudo se referem ao perfil sociodemográfico dos cuidadores, sua condição de saúde e cuidados prestados, em relação à capacidade de resiliência dos participantes.

Dos 101 idosos que fizeram parte da amostra, 84 eram mulheres, 58 estudaram até oito anos e 81 estavam acima dos 46 anos.

Através do CDR, 50 idosos apresentaram estadiamento 1 (demência leve), 44 apresentaram estadiamento 2 (demência moderada) e sete idosos com estadiamento 3 (demência grave), ou seja, mais da metade com possíveis complicações cognitivas e comportamentais. Em relação aos resultados do Mini Exame do Estado Mental (MEEM), apenas três idosos com demência leve não apresentaram prejuízo cognitivo.

Na tabela 1 observamos os dados de identificação, as características sociodemográficas e hábitos de vida dos cuidadores de idosos com doença de Alzheimer.

Tabela 1 - Dados de identificação, sociodemográficos e hábitos de vida, referentes aos cuidadores de idosos com doença de Alzheimer, Ribeirão Preto-SP, 2010

\begin{tabular}{llrr}
\hline $\begin{array}{l}\text { Dados de identificação, } \\
\text { sociodemográficos e hábitos } \\
\text { de vida }\end{array}$ & \multicolumn{2}{c}{ Cuidadores } \\
\cline { 2 - 4 } Gênero & M & $\%$ \\
Idade (anos) & $\mathrm{F}$ & 17 & 16,9 \\
& 18 a 45 & 84 & 83,1 \\
Estado civil & 46 ou mais & 20 & 19,8 \\
& Solteiro & 81 & 80,2 \\
& Casado & 9 & 8,9 \\
& Viúvo & 39 & 78,3 \\
& Divorciado/separado/ & 10 & 2,9 \\
Tempo de estudo & desquitado & & \\
(anos) & Analfabeto & 2 & 1,9 \\
& Até 8 & 58 & 57,5 \\
& De 9 a 11 & 30 & 29,8 \\
& Acima de 12 & 11 & 10,8
\end{tabular}




\begin{tabular}{llrr}
\hline $\begin{array}{l}\text { Professa uma } \\
\text { religião }\end{array}$ & Sim & 99 & 98,1 \\
& Não & 2 & 1,9 \\
Prática de & Não pratica & 69 & 68,4 \\
exercício físico & 1 vez/semana & 7 & 6,9 \\
& 2 vezes/semana & 11 & 10,9 \\
& 3 vezes/semana & 10 & 9,9 \\
& 4 vezes/semana ou & 4 & 3,9 \\
Reside com o & mais & & \\
idoso & Sim & 56 & 55,4 \\
& Não & 45 & 44,6 \\
\hline Total & & $\mathbf{1 0 1}$ & $\mathbf{1 0 0}$ \\
\hline
\end{tabular}

A maioria, 84 dos cuidadores era do sexo feminino, 81 estavam com 46 anos ou mais, sendo 37 maiores de 60 anos. Esse grupo de cuidadores era composto por maioria branca com 77 cuidadores, sendo 19 pardos, quatro negros e um de etnia amarela. Destes, 79 eram casados.

Observa-se que dois eram analfabetos, 58 cuidadores tinham até oito anos de estudo e 11 tinham de nove a onze anos de estudo.

A maioria dos cuidadores professava uma religião, dois referiram não ter religião e grande parte desses referiu ser praticante da religião informada.

Sessenta e nove cuidadores não realizavam nenhum exercício físico, sete praticavam pelo menos uma vez na semana, 11 praticavam duas vezes na semana, 10 três vezes e somente quatro cuidadores praticavam exercícios quatro vezes ou mais na semana.

Observa-se que metade dos cuidadores morava com o companheiro, filhos, sobrinho, genro e nora, 31 moravam apenas com o cônjuge, 13 moravam com pessoas de três gerações e seis faziam parte de arranjo intrageracional. Dos 101 cuidadores, 45 tinham até dois filhos, 47 tinham três ou mais e nove cuidadores não tinham filhos. Mais da metade da amostra, ou seja, 56 moravam na mesma residência que o idoso.

A maioria dos cuidadores afirmou realizar atividades de lazer, sendo que 81 se dedicam ao artesanato, crochê, assim como idas a cinemas, bailes, participação em viagens, passeios e churrascos com amigos.

Dos 95 cuidadores familiares, 48 eram filhos, seguidos de 32 cônjuges, nove cuidadores eram primos e noras, quatro eram irmãos e dois eram cunhados. Quarenta e seis cuidadores não trabalhavam e exerciam atividades do lar, 11 recebiam aposentadoria e permaneciam no lar, dois desenvolviam atividade autônoma fora do lar e 36 possuíam vínculo empregatício. Três cuidadores recebiam até um salário mínimo $(\mathrm{SM}), 58$ recebiam mais de um até três, 36 recebiam de quatro a 10 e apenas quatro cuidadores recebiam acima de 10 salários mínimos por mês.

Em relação à condição da saúde dos cuidadores associada à resiliência, observamos a tabela 2 .

Tabela 2 - Condição da saúde dos cuidadores de idosos com doença de Alzheimer associada à resiliência, Ribeirão Preto-SP, 2010

\begin{tabular}{|c|c|c|c|c|c|c|c|c|}
\hline \multirow{3}{*}{ Condição da saúde } & \multicolumn{6}{|c|}{ Grau de resiliência } & \multirow{3}{*}{ Total } & \multirow{3}{*}{ Teste de Fisher $(p)$} \\
\hline & \multicolumn{2}{|c|}{ Baixa } & \multicolumn{2}{|c|}{ Média } & \multicolumn{2}{|c|}{ Alta } & & \\
\hline & $\mathrm{n}$ & $\%$ & $n$ & $\%$ & $\mathrm{n}$ & $\%$ & & \\
\hline Tratamento médico & & & & & & & & \multirow{3}{*}{0,012} \\
\hline Sim & 22 & 43,1 & 12 & 23,5 & 17 & 33,3 & 51 & \\
\hline Não & 9 & 18 & 23 & 46 & 18 & 36 & 50 & \\
\hline \multicolumn{9}{|l|}{ Uso de medicamento } \\
\hline Sim & 24 & 40 & 18 & 30 & 18 & 30 & 60 & \multirow{2}{*}{0,043} \\
\hline Não & 7 & 17 & 17 & 41,4 & 17 & 41,4 & 41 & \\
\hline \multicolumn{9}{|l|}{ Cansaço } \\
\hline Sim & 26 & 36,6 & 28 & 39,4 & 17 & 23,9 & 71 & \multirow{2}{*}{0,003} \\
\hline Não & 5 & 16,6 & 7 & 23,3 & 18 & 60 & 30 & \\
\hline \multicolumn{9}{|l|}{ Esgotamento } \\
\hline Sim & 22 & 40 & 20 & 36,3 & 13 & 23,6 & 55 & \multirow{2}{*}{0,020} \\
\hline Não & 9 & 19,5 & 15 & 32,6 & 22 & 47,8 & 46 & \\
\hline \multicolumn{9}{|l|}{ Desânimo } \\
\hline Sim & 22 & 40 & 20 & 36,3 & 13 & 23,6 & 55 & \multirow{2}{*}{0,020} \\
\hline Não & 9 & 19,5 & 15 & 32,6 & 22 & 47,8 & 46 & \\
\hline
\end{tabular}


Cinquenta e um cuidadores referiram ter pelo menos, um problema de saúde com seguimento clínico e desses, quase metade apresentou baixo grau de resiliência com significância $(p=0,012) ; 60$ referiram usar algum tipo de medicamento alopata sendo que 42 deles apresentaram baixo e médio grau de resiliência com significância $(p=0,043)$. Quinze cuidadores referiram seguimento com psiquiatra e uso de antidepressivos. Dois cuidadores tiveram o diagnóstico e tratamento para depressão no passado.

Em relação às alterações ou queixas, 71 cuidadores referiram cansaço e 54 deles apresentaram baixo e médio grau de resiliência fortemente significante no teste de Fisher ( $\mathrm{p}=0,003) ; 55$ apresentaram esgotamento sendo 42 cuidadores com baixo e médio grau de resiliência com significância $(p=0,020)$ e 55 que apresentaram desânimo com 42 deles apresentando baixo e médio grau de resiliência e significância $(p=0,020)$.

Referente ao tipo de cuidador e preparo para o cuidar de idosos com doença de Alzheimer, observamos a tabela 3.

Tabela 3 - Tipo de cuidador e preparo para o cuidar de idosos com doença de Alzheimer, Ribeirão Preto-SP, 2010

\begin{tabular}{llrc}
\hline \multirow{2}{*}{ Tipo de cuidador } & \multicolumn{2}{c}{ Preparo } \\
\cline { 3 - 4 } & & $\mathbf{n}$ & $\%$ \\
\hline Cuidador familiar & Sim & 95 & 94 \\
Experiência anterior & Não & 6 & 5,9 \\
(cuidador) & Sim & 36 & 35,7 \\
Curso de formação & Não & 65 & 64,3 \\
& Sim completo & 14 & 13,9 \\
& Sim incompleto & 7 & 6,9 \\
Horas no cuidado & Não & 80 & 79,2 \\
(semanal) & Até 42 & 29 & 28,4 \\
& 43 a 71 & 16 & 15,6 \\
Ajuda para cuidar & S2 e mais & 56 & 55,3 \\
& Sim, de familiar & 47 & 46,5 \\
& Sim, de outra & 18 & 17,9 \\
Conhecimento sobre & pessoa & 36 & 35,6 \\
Alzheimer & São & 77 & 76,2 \\
\hline Total & Não & 24 & 23,8 \\
\hline
\end{tabular}

Dos 101 cuidadores, 95 eram familiares e seis exerciam essa atividade profissionalmente; 65 não tinham nenhuma experiência prévia de cuidado.

Quatorze cuidadores realizaram integralmente um curso oferecido pelo próprio hospital, sete cuidadores não o concluíram e 80 não tinham tido a oportunidade ou interesse em realizar curso de formação para cuidador.

O tempo dedicado ao cuidar era, para 29 cuidadores, de até 42 horas semanais, 16 cuidavam de 43 a 71 horas e 56 cuidavam de 72 horas e mais.

Dentre esses cuidadores, 47 dividiam a tarefa com outros familiares, 18 recebiam ajuda de vizinhos e amigos e 36 não recebiam nenhuma ajuda nessa tarefa.

Em relação ao conhecimento sobre a doença de Alzheimer, sua evolução e estadiamentos, 77 cuidadores afirmaram ter conhecimento.

Dentre os cuidados realizados pelos cuidadores destacaram-se as supervisões e auxílios na administração de medicações, alimentação, higienização e acompanhamentos em retornos médicos.

\section{Associações entre as características do cui- dador e o grau de resiliência}

Foram analisadas as associações entre as características do cuidador e grau de resiliência que indicaram associação significante com o teste de Fisher. Os dados serão mostrados na tabela 4.

Merece destaque o grau de parentesco e sua relação com o grau de resiliência, fortemente significante no teste exato de Fisher $(p=0,005)$. Filhos e cônjuges apresentaram médio e alto grau de resiliência, quando comparados a irmãos e cunhados. Os outros cuidadores, como primos e noras, tiveram alto e médio grau de resiliência, respectivamente.

Em relação ao desânimo, 42 cuidadores apresentaram associação significante $(p=0,020)$ com alto e médio grau de resiliência; para a alteração na atividade sexual, quase metade dos cuidadores apresentaram baixa resiliência com significância $(p=0,046)$ e referente ao uso de medicamento, 42 cuidadores apresentaram baixo e médio grau de resiliência com significância $(\mathrm{p}=0,043)$. 
Tabela 4 - Associação entre características do cuidador e grau de resiliência, Ribeirão Preto-SP, 2010

\begin{tabular}{|c|c|c|c|c|c|c|c|c|}
\hline \multirow{3}{*}{ Características do cuidador } & \multicolumn{6}{|c|}{ Grau de resiliência } & \multirow{3}{*}{ Total } & \multirow{3}{*}{ Teste de Fisher (p) } \\
\hline & \multicolumn{2}{|c|}{ Baixa } & \multicolumn{2}{|c|}{ Média } & \multicolumn{2}{|c|}{ Alta } & & \\
\hline & $\mathrm{n}$ & $\%$ & $\mathrm{n}$ & $\%$ & $\mathrm{n}$ & $\%$ & & \\
\hline Parentesco & & & & & & & & \\
\hline Filhos(as) & 17 & 35,4 & 12 & 25 & 19 & 39,5 & 48 & \multirow{6}{*}{0,005} \\
\hline Cônjuge & 10 & 31,3 & 17 & 53,2 & 5 & 15,6 & 32 & \\
\hline Irmão(a) & 2 & 50 & 1 & 25 & 1 & 25 & 4 & \\
\hline Cunhado(a) & 1 & 50 & 0 & 0 & 1 & 50 & 2 & \\
\hline Outros & 0 & 0 & 5 & 55,5 & 4 & 44,5 & 9 & \\
\hline Não se aplica & 1 & 16,7 & 0 & 0 & 5 & 83,3 & 6 & \\
\hline \multicolumn{9}{|l|}{ Desânimo } \\
\hline Sim & 22 & 40 & 20 & 36,3 & 13 & 23,6 & 55 & \multirow[t]{2}{*}{0,020} \\
\hline Não & 9 & 19,5 & 15 & 32,6 & 22 & 47,8 & 46 & \\
\hline \multicolumn{9}{|l|}{ Alteração atividade sexual } \\
\hline Sim & 15 & 37,5 & 17 & 42,5 & 8 & 20 & 40 & \multirow{2}{*}{0,046} \\
\hline Não & 16 & 26,3 & 18 & 29,5 & 27 & 44,3 & 61 & \\
\hline \multicolumn{9}{|l|}{ Uso de medicamento } \\
\hline Sim & 24 & 40 & 18 & 30 & 18 & 30 & 60 & \multirow{2}{*}{0,043} \\
\hline Não & 7 & 17 & 17 & 41,4 & 17 & 41,4 & 41 & \\
\hline
\end{tabular}

\section{DISCUSSÃO}

A maioria dos idosos cuidados era do sexo feminino, o que corrobora os resultados de estudo epidemiológico sobre demência na população idosa. $^{9}$

A situação é desafiadora para a área da saúde, pois, à medida que envelhece mais dependente o idoso se torna para as atividades da vida diária. Esse fato é agravado pela ocorrência de outras doenças crônicas que requerem cuidados específicos. Essas pessoas se tornam cada vez mais dependentes de cuidados com diferentes graus de comprometimento, perdas e necessidades. ${ }^{10}$

Vale destacar que 82 idosos deste estudo apresentavam prejuízos cognitivos graves ao serem avaliados pelo MEEM. Na avaliação do CDR, que verifica as influências dessas perdas cognitivas na capacidade para realizar adequadamente as atividades da vida diária, 52 idosos apresentavam maior prejuízo, indicando que alguns, mesmo tendo prejuízos cognitivos graves, naquele momento, necessariamente, não estavam precisando de auxílio para determinadas tarefas. No entanto, a evolução da doença ocorre e, certamente, os cuidadores, em algum momento, irão ampliar suas tarefas para o cuidado.

A maioria dos cuidadores era familiar, que não exercia atividade profissional remunerada, sem experiência prévia de cuidado e não realizou o curso de formação. Apesar disso, a tarefa de cuidar pode trazer benefícios ou resultados positivos, tais como satisfação pessoal, melhora no senso de realização, aumento do sentimento de orgulho e habilidade para enfrentar novos desafios, melhora no relacionamento com o idoso, sentimento de retribuição, entre outros. Entretanto, o que pode prevalecer, muitas vezes, é a sobrecarga ou o ônus decorrente do estresse emocional, do desgaste físico, de problemas de saúde, das limitações para as atividades de trabalho, lazer e vida social, além dos conflitos familiares, incertezas e insegurança quanto ao trabalho realizado e à proximidade da morte do idoso. ${ }^{11}$

Nenhum cuidador atingiu o escore para depressão no rastreamento deste estudo. Mesmo não pontuando, um cuidador referiu ter diagnóstico de transtorno mental, com uso de antidepressivo e antipsicótico e oito cuidadores queixaram-se de esquecimento e demonstraram preocupação com esse fato.

Interessante destacar que dos seis cuidadores profissionais, cinco pontuaram alto grau de resiliência.

Para os cuidadores com experiência de cuidado com mais de dois anos, as atividades foram se tornando desgastantes. Esses enfatizaram o abalo do seu estado emocional, aliado ao cansaço físico. Além disso, a finitude passa a ser preocupação mais concreta, gera angústia para os cuidadores por considerarem que, dali a algum tempo, poderão não estar mais com o idoso. Os cuidadores que estão há mais de 10 anos na atividade 
de cuidado ao idoso, atualmente, recebem ajuda de outras pessoas e referiram desgaste emocional e insônia, pois o idoso já apresenta comprometimento cognitivo grave. Os idosos que são cuidados há mais de 18 anos estão acamados, necessitam de auxílio para todas as atividades e seus cuidadores demonstraram reconhecer que essa fase é a mais difícil e por isso exige maior dedicação.

Aproximadamente $40 \%$ da amostra têm mais de 60 anos o que indica que idosos estão cuidando de idosos. Estudo internacional sobre idosos dependentes constatou que $59 \%$ dos cuidadores tinham acima de 50 anos e $36 \%$ tinham mais de 65 anos. Os dados mostraram também que 45\% dos cuidadores eram idosos, com faixa etária entre 65 e 85 anos, que cuidavam de $60 \%$ de pacientes também idosos. ${ }^{12}$

Alguns cuidadores do presente estudo relataram, em decorrência da idade, queixas de esquecimento, dificuldade auditiva e dificuldade motora. Essas ocorrências preocupam os cuidadores, pois são responsáveis pelo cuidado e, na maior parte do tempo, ficam sozinhos em casa com os idosos. O grande desafio é lidar com o surgimento das doenças no cuidador, pelo fato de estarem envelhecendo, o que justifica a necessidade da colaboração de outras pessoas para manter o cuidado.

Até recentemente, no Brasil, a população de idosos era pequena e a família assumia o cuidado no lar, exceto nas situações clínicas ou cirúrgicas que necessitavam de internação em hospitais gerais. Esse panorama tem sofrido alterações nos últimos anos. Existe preocupação recente relacionada ao esclarecimento sobre as demências, sua evolução e tratamento, associada ao atendimento de pacientes e famílias. A falta de conhecimento específico pode favorecer situações de risco no cuidado e nas relações entre idoso e cuidador, como, por exemplo, violência doméstica. ${ }^{13}$

Quase todos os cuidadores referiram possuir algum conhecimento sobre a doença de Alzheimer. O fato de terem experiência prévia e possuírem conhecimento básico sobre a doença pode influenciar a aceitação da condição de ser cuidador.

A maioria dos questionamentos dos cuidadores estava relacionada com o cuidado propriamente dito, principalmente sobre as condutas corretas em determinadas tarefas e na abordagem relacional, já que o idoso apresenta alterações comportamentais e de linguagem.

Conflitos familiares intergeracionais podem surgir em decorrência da responsabilidade nas atividades do cuidar, relativas à dependência do idoso e pelo fato de se sentirem sobrecarregados. ${ }^{14}$ $\mathrm{Na}$ amostra pesquisada, houve referências a situações estressantes no dia a dia do cuidador, porém, na maioria dos casos, esses cuidadores mostraram-se tranquilos e souberam lidar com a situação, com a ajuda de outra pessoa. Mais da metade dos casos recebia ajuda de outras pessoas na tarefa de cuidar, o que pode ser reconhecido como aspecto amortecedor das dificuldades do dia a dia.

O familiar cuidador, ao se dedicar integralmente ao idoso com doença de Alzheimer, pode se tornar estressado e cansado, física e mentalmente, o que tende a piorar se ele estiver sozinho, sem a ajuda de outras pessoas. ${ }^{15}$

A ajuda recebida de outros membros da família, de amigos e de profissionais é igualmente importante para o desempenho adequado do seu papel, como também para fortalecer o sentimento de apoio e envolvimento.

Não houve pontuação para depressão na amostra, o que corrobora o estudo ${ }^{19}$, em que elevada resiliência foi correlacionada a baixa somatização e depressão, bem como com melhor saúde física, determinação, sensação de bem-estar e habilidade para lidar com problemas. Contudo, em estudo norte-americano encontrou-se associação entre cuidadores com altos níveis de depressão e idosos com alto nível de prejuízo cognitivo. ${ }^{16}$

A maioria professava uma religião, em seus relatos, fizeram questão de enfatizar o quanto se sentem fortes por terem fé em Deus, fato que os está ajudando na tarefa do cuidado ao idoso demenciado, inclusive com a proximidade da morte biológica e a finitude do ser. As crenças em um ser superior, a fé, a espiritualidade e as práticas religiosas são percebidas pelos cuidadores como estratégias muito eficazes de enfrentamento da sobrecarga de cuidado, angústia, estresse e depressão. ${ }^{17}$

\section{CONCLUSÕES}

As condições sociodemográficas, hábitos de vida e condição de saúde influenciam na capacidade de resiliência do cuidador.

Apesar das dificuldades para cuidar de idosos com doença de Alzheimer, os cuidadores conseguem realizar essa atividade com mais eficácia quando possuem conhecimento sobre a doença e recebem ajuda de outras pessoas.

Neste estudo focalizaram-se os cuidadores que acompanhavam idosos para atendimento em hospital público e uma unidade básica de saúde 
e que exerciam essa função há mais de um ano. Necessário se faz a investigação em outros contextos da atenção em saúde como ambulatórios, serviços privados e outras unidades de atenção básica, onde muitos idosos podem ser diagnosticados com essa doença e a família necessita suporte profissional.

O enfermeiro pode atuar enquanto cuidador direto, bem como educador e dirigir seus conhecimentos tanto para os idosos como para os cuidadores, nos diferentes contextos de atenção à saúde do idoso. Em relação ao papel preventivo, pode identificar as manifestações da demência e alertar a pessoa e sua família para a busca de orientações e cuidados necessários.

O idoso com doença de Alzheimer, em seu contexto familiar e social, poderá ter melhores condições de saúde e qualidade de vida, se o cuidador tiver maior capacidade resiliente, pois este mantém o seu equilíbrio mental e físico, o que favorece a realização da sua tarefa de cuidar.

É importante conhecer o grau de escolaridade, tanto do cuidador quanto do idoso, quando se pretende abordagem educativa pelo enfermeiro. Além disso, deve-se procurar identificar primeiramente as reais necessidades de cada indivíduo, conhecer a fase da doença do idoso e quais as possibilidades do cuidador e da enfermagem em relação à família. Esse "diagnóstico" sobre o cuidador, realizado pelo enfermeiro, poderá trazer informações valiosas para promover sua própria capacidade resiliente.

\section{REFERÊNCIAS}

1. Instituto Brasileiro de Geografia e Estatística. Censo demográfico de 2010 - Resultado do universo. [Acesso 2011 Nov 05] Disponível em: http:// www.ibge.gov.br/home/estatistica/população/ censo2010/tabela

2. Rutter M. Resilience: some conceptual considerations. J Adolesc Health. 1993; 14: 626-31.

3. Sória DAC, Souza IEO, Moreira M C, Santoro DC, Menezes MFB, Moreira MCA. Resiliência como objeto de Investigação na Enfermagem e em outras áreas: uma revisão. Esc Anna Nery Rev Enferm. 2006 Dez; 10(3):547-51.

4. Ahern NR, Kiehl EM, Sole ML. A rewiew of instruments measuring resilience. Iss Compr Pediatr Nurs. 2006 Apr-Jun; 29(2):103-25.

Correspondência: Cheila Cristina Leonardo de Oliveira Gaioli Rua Laguna, 861

14090-062 - Jardim Paulista, Ribeirão Preto, SP, Brasil

E-mail: cheila@eerp.usp.br
5. Arruda MC, Alvarez AM, Gonçalves LHT. O familiar cuidador de portador de doença de Alzheimer participante de um grupo de ajuda mútua. Ciênc Cuid Saúde. 2008 Jul-Set; 7(3):339-45.

6. Gorenstein NC, Andrade L. Inventário de depressão de Beck: propriedades psicométricas da versão em português. Rev de Psiq Clin 1998; 25(5):245-50.

7. Wagnild GM, Young HM. Development and psychometric evaluation of resilience scale. J Nurs Measur 1993 Winter; 1:165-78.

8. Pesce RP, Assis SG, Avanci JQ, Santos NC, Malaquias JV, Carvalhaes R. Adaptação transcultural, confiabilidade e validade da escala de resiliência. Cad Saúde Pública 2005 Mar- Apr; 21(2):436-48.

9. Fagundes SD, Prevalência de demência entre os idosos brasileiros: revisão sistemática [dissertação]. Brasília (DF): Universidade Católica de Brasília; 2008.

10. Inouye K. Educação, qualidade de vida e doença de Alzheimer: visões de idosos e seus familiares [dissertação]. São Carlos (SP): Universidade Federal de São Carlos; 2008.

11. Gaioli CCLO. Cuidadores de idosos com doença de Alzheimer: variáveis sociodemográficas e da saúde associadas à resiliência [tese] Ribeirão Preto (SP): Universidade de São Paulo Escola de Enfermagem de Ribeirão Preto; 2010.

12. Alzheimer's Association. 2008 Alzheimer's disease facts and figures. Alzheimer Dement. 2008 Mar; 4(2):110-33.

13. Santana RF, Almeida KS, Savoldi NAM. Indicativos de aplicabilidade das orientações de enfermagem no cotidiano de cuidadores de portadores de Alzheimer. Rev Esc Enferm USP. 2009 Jun; 43(2):459-64.

14. Fialho PPA, Koenig AM, Santos EL, Guimarães HC, Beato RG, Carvalho VA. Dementia caregiver burden in a brazilian sample: association to neuropsychiatric symptoms. Dementia Neuropsych. 2009 Jun; 3(2):132-5.

15. Ricci M, Guidoni SV, Sepe-Monti M, Bomboi G, Antonini G, Blundo C. Clinical findings, functional abilities and caregiver distress in the early stage of dementia with Lewy bodies (DLB) and Alzheimer's disease (AD). Arch Gerontol Geriatr. 2009 SepOct;49(2):e101-4.

16. Zautra AJ, Hall JS, Murray KE. Resilience: a new integrative approach to health and mental health research. Health Psych Rew. 2008; 2(1):41-64.

17. Paula J, Roque F, Araújo F. Qualidade de vida em cuidadores de idosos portadores de demência de Alzheimer. J Bras Psiq. 2008; 57(4):283-7.

Recebido: 8 de novembro de 2010 Aprovado: 15 de setembro de 2011 